\title{
Preparation of Black Poly(Methyl Methacrylate-Ethyleneglycol Dimethacrylate) Microspheres Using Metallic Complex Dyes
}

\author{
Shen-Kung Liao*, Shin-Bin Wu, Chie-Hao Chao \\ Department of Fiber and Composite Materials, Feng Chia University, Taiwan \\ Email: *skliao@fcu.edu.tw
}

How to cite this paper: Liao, S.-K., Wu, S.-B. and Chao, C.-H. (2020) Preparation of Black Poly(Methyl Methacrylate-Ethyleneglycol Dimethacrylate) Microspheres Using Metallic Complex Dyes. Materials Sciences and Applications, 11, 692-713.

https://doi.org/10.4236/msa.2020.1110047

Received: September 22, 2020

Accepted: October 27, 2020

Published: October 30, 2020

Copyright $\odot 2020$ by author(s) and Scientific Research Publishing Inc. This work is licensed under the Creative Commons Attribution International License (CC BY 4.0).

http://creativecommons.org/licenses/by/4.0/

\begin{abstract}
In this study, we synthesized polymethyl methacrylate cross-linked microspheres and functionalized the surface of the microspheres with amine groups, and discussed the effect of functionalization and the change of the surface morphology of the microspheres. This research uses 1:2 metal composite acid dye to dye self-synthesized microspheres with uniform particle size, and successfully prepares black polymethyl methacrylate cross-linked microspheres, which are applied to spacer microspheres.
\end{abstract}

\section{Keywords}

PMMA, Microsphere, Metallic Complex Dye, Crosslinked

\section{Introduction}

A polymer sphere is a substance in which a plurality of polymer chains are wound and stacked on each other and are present in a spherical structure. Polymer spheres with different compositions, particle sizes, appearances and structures have their own unique properties and can be widely used in daily life. The preparation methods of polymer microspheres are shown in Figure 1, and the synthetic raw materials can be roughly distinguished. There are two types, one is a polymerization reaction or a polycondensation reaction starting from a monomer to synthesize microspheres, and the other is a polymer solution which is treated by physical or chemical means to form microspheres (particles).

The LCD screen is mixed with the "spacer microspheres" in the liquid crystal and "standing" between the glass panels. It mainly functions as a "skeleton" and is placed between two substrates filled with liquid crystal material to provide 
support for the liquid crystal cell. The gap can precisely control the thickness of the glass substrate and the liquid crystal layer. Since the liquid crystal layer has a uniform thickness and a load-bearing capacity, the spacer polymer microspheres need a high particle size uniformity, and the average particle diameter is between 3 and 7 micrometers. It has elasticity, high mechanical strength, smooth surface, high cleanliness and extremely low metal impurities. Therefore, the commonly used spacers are rod-shaped and spherical, mainly spherical, and the materials are made of polymer and glass. The most common, the glass material is cheaper, the particle size is easy to control, but it is easy to scratch the glass substrate or the electrode; the polymer material is soft, the particle size uniformity is not easy to control, and when the material is shaken, it is easy to cause the movement of the microsphere (Table 1 ). It is a comparison of the uniformity of the particle size of the microspheres, but the application process of the polymer sphere is simple and is the mainstream product on the market. Today, the LCD industry

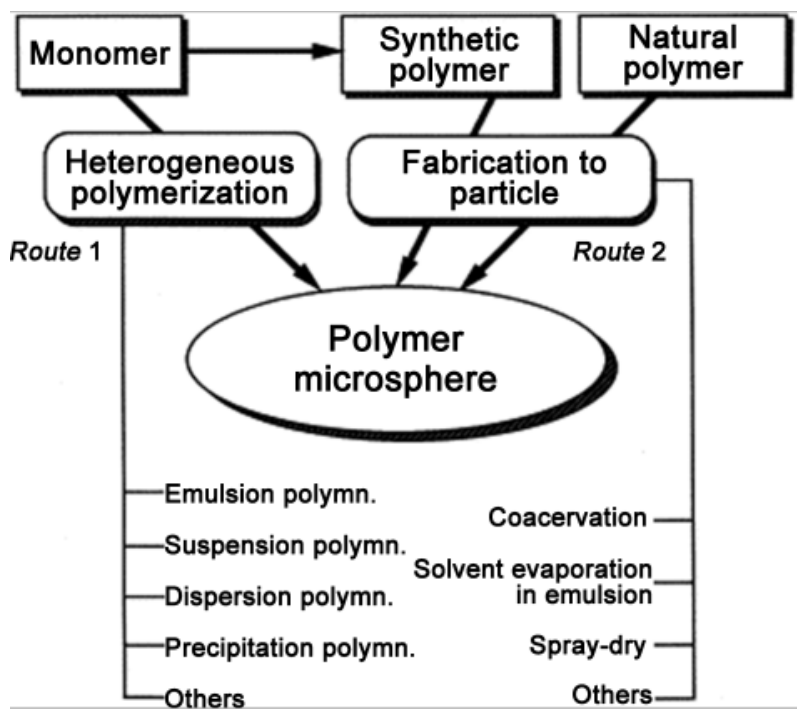

Figure 1. Preparation of polymer spheres [1].

Table 1. Comparison of particle size uniformity effects of microspheres.

\begin{tabular}{|lll|}
\hline \multicolumn{1}{|c|}{$\begin{array}{l}\text { Microspheres have a narrow particle size } \\
\text { distribution }\end{array}$} & $\begin{array}{l}\text { Microspheres have a wide particle size } \\
\text { distribution }\end{array}$ \\
\hline $\begin{array}{l}\text { schematic } \\
\text { diagram }\end{array}$ & Suppress the movement of \\
microspheres & Easy to cause microsphere movement \\
\hline $\begin{array}{l}\text { When } \\
\text { shaking }\end{array}$ & $\begin{array}{l}\text { a small number of microspheres } \\
\text { can control the interval }\end{array}$ & $\begin{array}{l}\text { Uneven particle size } \\
\text { Need a lot of microspheres to effectively } \\
\text { control the interval }\end{array}$ \\
\hline $\begin{array}{l}\text { Number of } \\
\text { microspheres }\end{array}$ & \\
\hline
\end{tabular}


is highly competitive. As the world's leading producer of liquid crystal displays, mastering key technologies is the first to maintain its leading position in the industry. Interstitial materials are indispensable in the liquid crystal industry and must conform to uniform particle size and stability. The rigorous conditions of physical and chemical properties are a key core technology.

Polymethyl methacrylate with excellent transparency and chemical stability is the current mainstream spacer microsphere material, and reference functionalized microsphere literature. Figure 2 shows that in 2008, Hong-Bo Liu [2] and other scholars used non-emulsion polymerization to prepare four different sizes of P (MMA/GMA/DVB) composite microspheres, and used ethylenediamine to open the epoxy groups on the surface of the microspheres and to achieve the purpose of surface functionalization of the microspheres. The results show that with the increase of the number of grafted layers, the number of amine groups increases, and the larger the volume of microspheres is, the better the grafting efficiency will be due to the reduction of steric hindrance.

According to the literature on microsphere dyeing, Figure 3 shows that in 2013, Hsien-Tsung Wu [3] and other scholars prepared PMMA colored microspheres by dispersion polymerization of green pigments. The results showed that the pigment and monomer were simultaneously polymerized and dispersed. The addition of water to the PMMA microspheres can produce small microspheres with uniform particle size, but the dispersion effect of water on the pigment causes a serious influence on the uniformity of the particle size of the microspheres. The ratio of the reactive monomer to the pigment also affects the product. The particle size distribution, while using ultrasonic waves, can effectively prevent the problem of pigment settling and delamination of the mixture during polymerization.
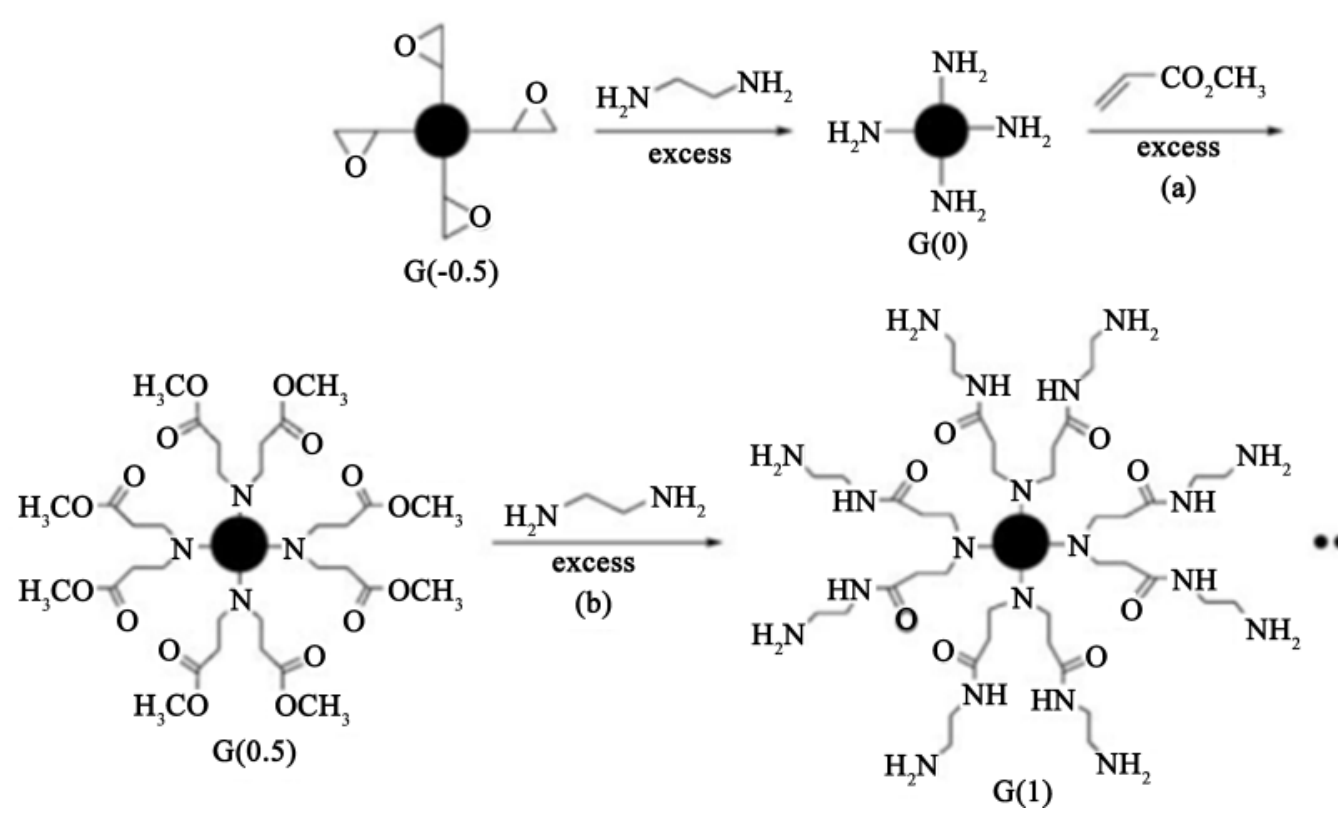

Figure 2. Schematic diagram of PAMAM synthesis of grafted dendritic tree on PMGD microspheres [2]. 
(a)

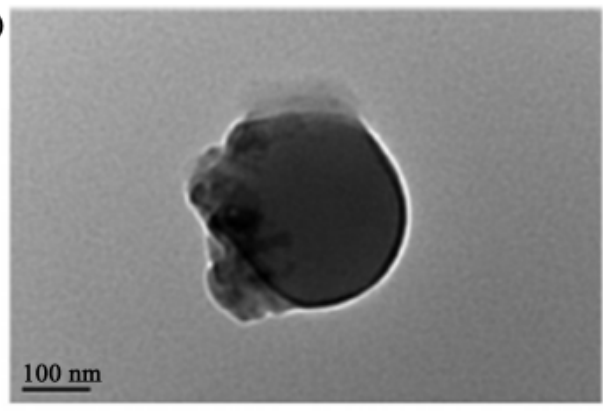

(c)

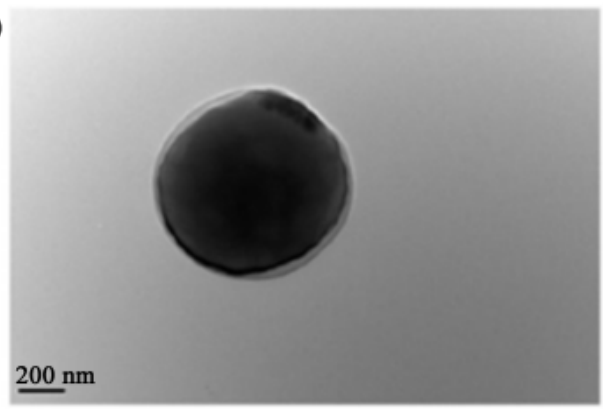

(b)

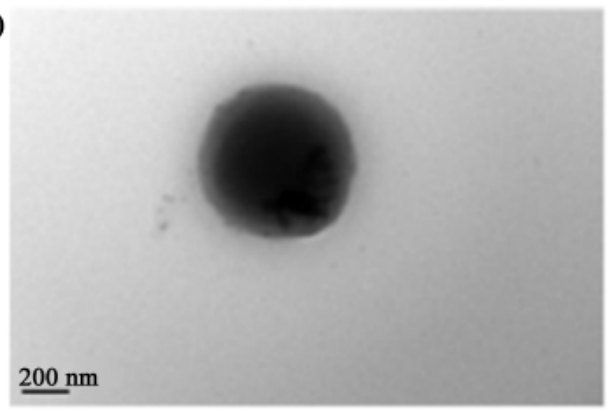

(d)

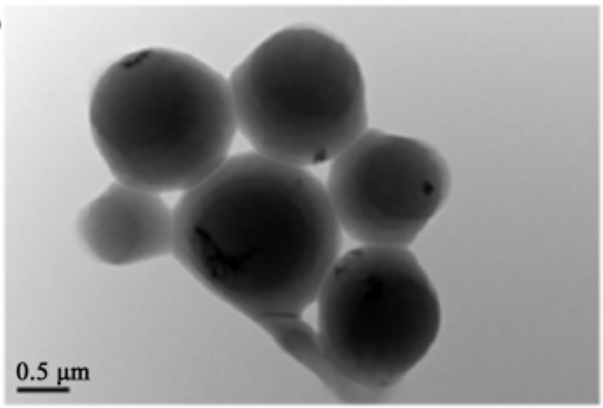

(e)

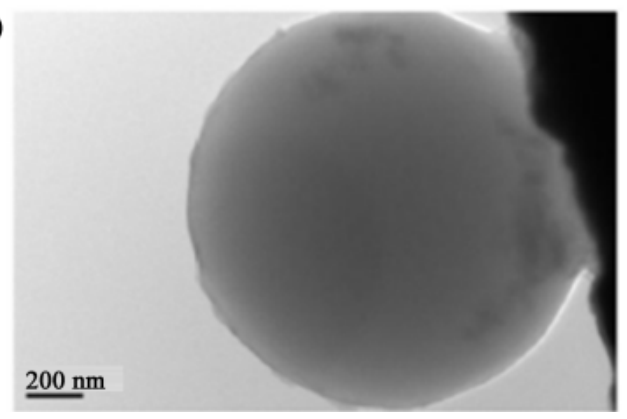

Figure 3. TEM image of composite particles prepared with different mass ratios (monomer/pigment) [3].

Compared with white microspheres, black microspheres with high absorbance can effectively reduce the occurrence of halo, improve color contrast, and have excellent imaging results. However, polymethyl methacrylate is an amorphous structure, and dyeing processing technology is difficult. Methyl methacrylate is easily eroded by alkali. In the related literature, most of the colored microspheres are prepared by embedding or dispersing in the microspheres, so that there is a problem of insufficient color depth and poor fastness. The absorbance effect of the spacer microspheres is limited.

In this study, P (MMA-EGDMA) crosslinked microspheres were synthesized by dispersion polymerization method, and the surface amine groups were functionalized. The three primary color metal complex dyes were used to dye and dye the microspheres to prepare black polymethyl methacrylate microspheres. It is expected that the high absorbance characteristic of the spacer material can effectively reduce the halo phenomenon. The ionic bond is formed between the crosslinked microspheres with functional groups and the metal complex acid dye, which overcomes the difficulty of dyeing, poor dye fastness and insufficient 
color depth. The problem is that the crosslinked structure gives the microspheres excellent mechanical properties.

\section{Experiment}

Figure 4 shows that the dyed microspheres of the functional dyeing of the experiment. The monomeric MMA and the stabilizer PVP are dissolved in $300 \mathrm{~mL}$ of the dispersion medium (Methanol/DI water) by referring to the method for preparing the functionalized microspheres [4] [5] [6]. In the middle, the initiator AIBN and the cross-linking agent EGDMA were mixed with $10 \mathrm{~mL}$ of methanol, respectively, and the three mixed solutions were ultrasonically shaken for 20 minutes, and the MMA mixed solution was placed in a $500 \mathrm{~mL}$ four-necked round bottom flask equipped with a condenser tube, AIBN and The EGDMA solution was separately added to a $200 \mathrm{~mL}$ separatory funnel. The flask was placed in an oil bath on a magnet mixer, and heating was started. After the temperature reached a set value of $60^{\circ} \mathrm{C}$, AIBN was slowly dropped into the flask to start polymerization, and the polymerization was carried out. After $12-72$ hours, after the reaction is completed, the reaction solution is removed by centrifugation, and the sample is removed by washing with methanol and deionized water to remove the stabilizer and residual monomers, and dried in an oven at $60^{\circ} \mathrm{C}$ for 24 hours to obtain the experiment at this stage.

Next, the microsphere surface modification functionalization process was carried out. $1 \mathrm{~g}$ of microsphere powder was mixed with $10 \mathrm{~mL}$ of methanol, and ultrasonically oscillated for 20 minutes to uniformly disperse it. The hexamethylenediamine was dissolved in DMSO, and the mixture was diluted into different moiré. The concentration of the modification solution, the microsphere solution and $5 \mathrm{~mL}$ of the amine modification solution were placed in a round neck flask, heated to reflux, cooled, and the reaction solution was removed, and washed with deionized water and methanol, followed by drying to obtain a functionalized micro Ball FP (MMA-EGDMA). After the microsphere dyeing operation, 1 g of FP (MMA-EGDMA) microspheres and $10 \mathrm{~mL}$ of ethanol were uniformly dispersed by ultrasonic vibration during the dyeing process, and the three dyes

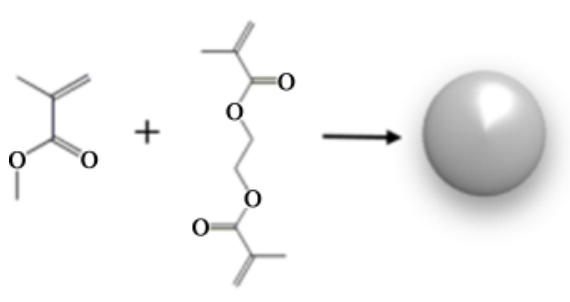

MMA

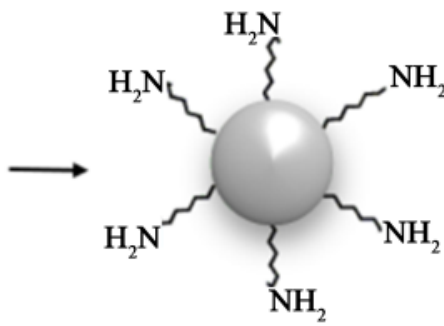

Functionalized Microsphere

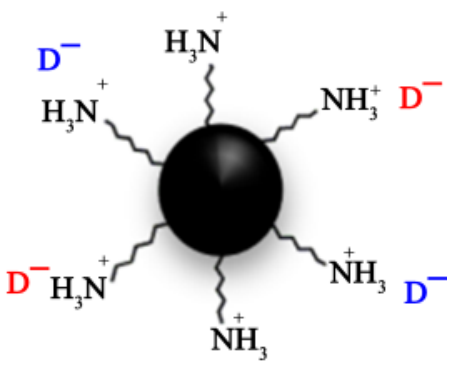

Black Microsphere

Figure 4. The sample P (MMA-EGDMA) microspheres. Experimental main architecture. 
were arranged in a ratio of $1: 1: 1$ with $20 \mathrm{~mL}$ of deionized water. The dyeing solution is in the dyeing bath, the initial temperature is 40 degrees, the heating rate is 1 degree by 1 minute, and after reaching 120 degrees, the temperature is maintained for 60 minutes, and the temperature is lowered to 40 degrees. After the dyeing is completed, it is repeatedly centrifuged and washed with deionized water, placed in an oven for drying, and subjected to subsequent evaluation and evaluation, and the effects of $\mathrm{pH}$ value and temperature on the dyeing performance are sequentially discussed.

Figure 5 shows the experimental process and sequence of this study.

\section{Results and Discussion}

In the preliminary experiment, the cross-linking EGDMA and the monomer MMA were simultaneously dispersed and polymerized, and the microsphere particles could not be prepared. Therefore, in the process of synthesizing the crosslinked polymer microspheres in one step, in order to avoid the sensitive initial nucleation stage. The granule flocculation is caused by the addition of the copolymer cross-linking agent cannot even nucleate. Therefore, EGDMA is added 1 to 4 hours after the start of the polymerization reaction. Figure 6 shows the different initial addition times of EGDMA [6] [7] [8]. P (MMA-EGDMA) microspheres FE-SEM analysis of the image, as can be seen from the figure, as the initial addition time shifts back, the microspheres tend to be smoother spherical, Figure 6(a) adds crosslinks after 1 hour. The growth of the microspheres of the agent is not complete. It can be observed that three mainly spherical particles adhere to each other and do not disperse to form a single spherical particle, which is composed of numerous nano-sized small particles, and many pores. In the presence of the sphere, the sphere is loose, because the monomer has not grown to a certain extent after the formation of the primary nucleus, and its properties are biased toward the oligomer which is soluble in the medium, and

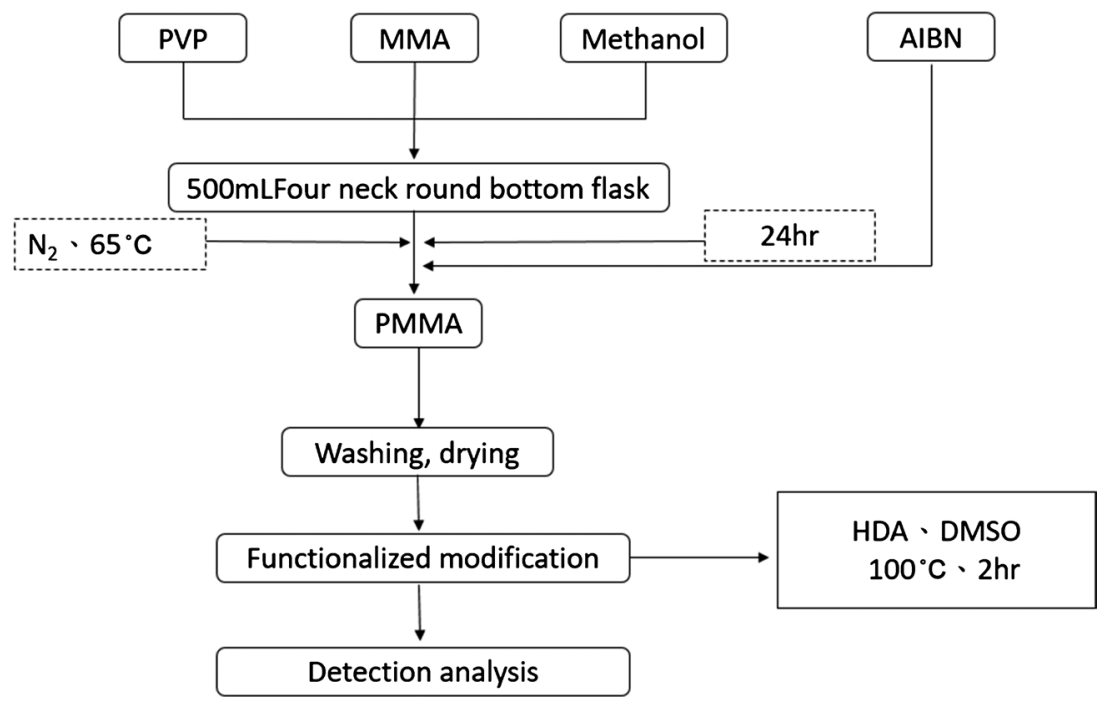

Figure 5. Experiment process. 


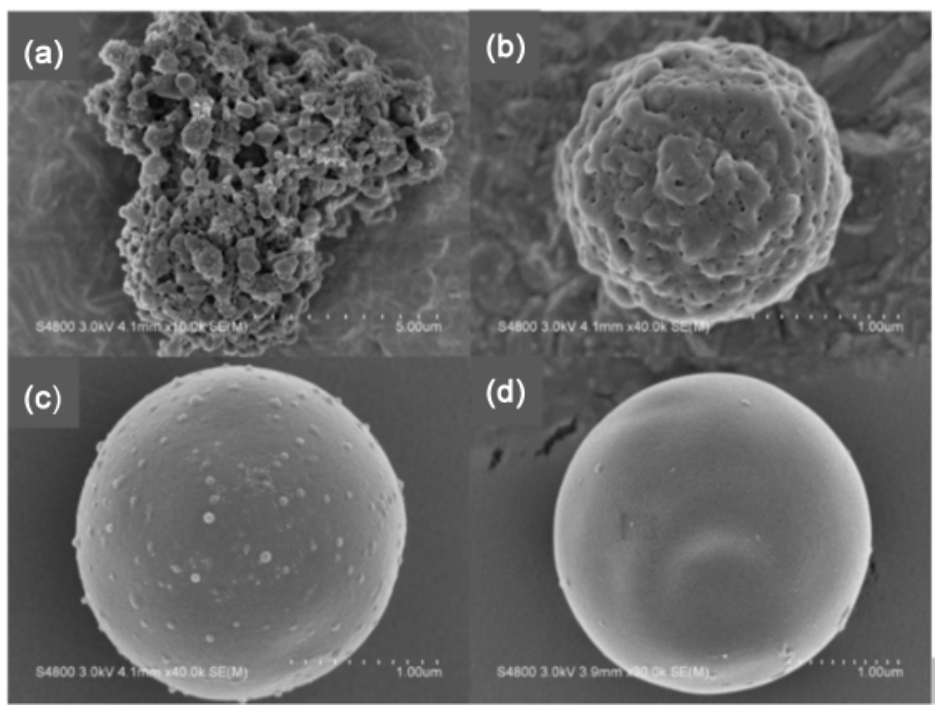

Figure 6. Cross-linked microspheres FE-SEM image of the initial addition time of the cross-linking agent. (a) $1 \mathrm{hr}$; (b) $2 \mathrm{hr}$; (c) $3 \mathrm{hr}$; (d) $4 \mathrm{hr}$.

the stability in the system is not sufficient. The addition of the crosslinker at the moment destroyed. The stability of a system and the ability to inhibit the growth of stable nuclei result in Sticky each other between the microspheres.

The independent single microspheres can be observed after prolonging the initial addition time to 2 - 4 hours. Figure $6(\mathrm{~b})$ shows the microspheres added with the cross-linking agent 2 hours after the start of the polymerization. The surface is uneven and there are still holes. However, the number and size of the holes are greatly reduced. Figure 6(c) and Figure 6(d) are microspheres with cross-linking agent added after 3 and 4 hours respectively. The surface of the cross-linked microspheres is not even porous. Secondary nucleation grains generated by liquid shear force, mutual collision between microspheres or collision of microspheres with the wall of the polymerization tank are adsorbed on the surface of the microspheres, and after a time of 4 hours, the surface is twice The number of crystal nucleation particles can be effectively reduced, the size is also reduced, and the prepared microspheres are smooth and compact.

\subsection{Solvent Ratio}

The initial addition time of the comonomer EGDMA [9] was set to 4 hours, and the monomer was fixed with experimental parameters such as other drugs, and only the proportion of the mixed solvent was changed. In Figure 7 The FE-SEM image of crosslinked microspheres prepared by using four different solvents (methanol/deionized water) as the polymerization medium, respectively, can be observed from Figure 7(a) when the proportion of mixed solvent is 10/0. The surface of the sphere is tight and slightly small particles adhere. As the content of deionized water increases, the polarity of the polymerization medium increases, and the polymerization rate also increases. When the ratio of the mixed solvent (methanol/deionized water) is $9 / 1$, Due to the increased polarity of the 


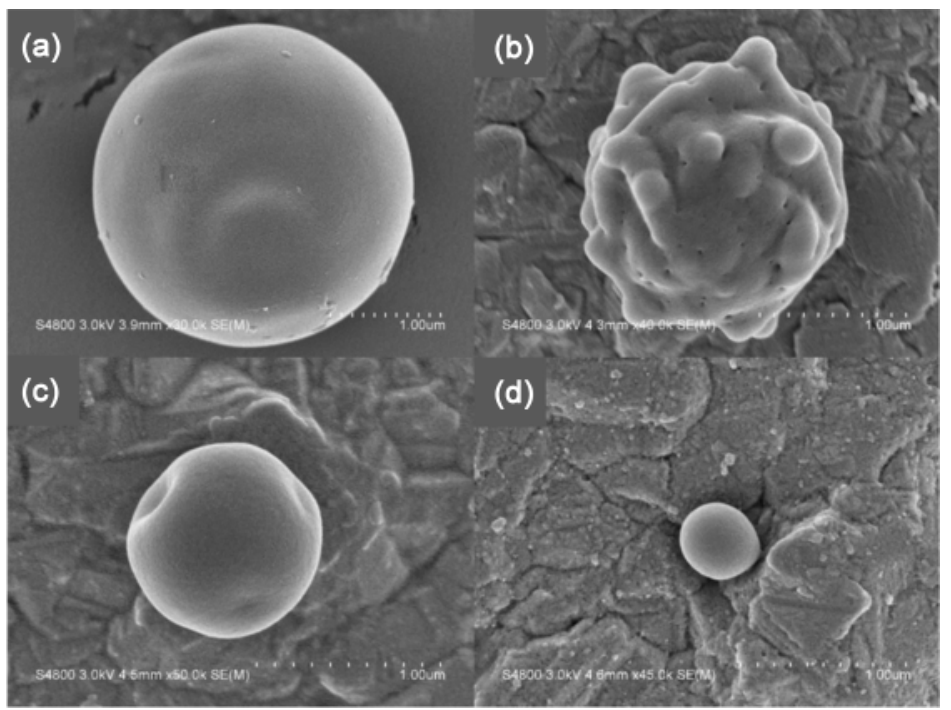

Figure 7. FE-SEM image of crosslinked microspheres prepared by ratio of different solvents (methanol/deionized water) (a) $10 / 0$; (b) $9 / 1$; (c) $8 / 2$; (d) $7 / 3$.

reaction medium, the monomer MMA cannot be fully absorbed into the microspheres for growth, resulting in the appearance of irregular spherical protrusions and voids on the surface of the microspheres, as shown in Figure $7(\mathrm{~b})$; The ratio of methanol/deionized water is $8 / 2$, and the surface of the synthesized crosslinked microspheres is clean. However, due to the stress phenomenon caused by the high crosslink density on the surface of the microspheres, the shape of the microspheres is deformed by depression Figure 7 (d). Very few microspheres that are fully protected by PVP are protected from condensation caused by phase separation.

\subsection{EGDMA Addition Amount}

In this stage, the methanol/deionized water ratios of $10 / 0$ and $9 / 1$ were fixed respectively. It is necessary to observe the change of microspheres with different concentrations of EGDMA to find the most suitable polymerization parameters, and the standard parameters of the most subsequent experimental steps.

First, the ratio of the polymerization medium (methanol/deionized water) was set to 10/0. Figure 8 shows the FE-SEM image of the crosslinked microspheres prepared by four different EGDMA concentrations. The observation pattern Figure 8(a) was not added any The PMMA microspheres of the EGMDA have a large number of protrusions and micropores on the surface of the sphere. As shown in Figure 8(b)), Figure 8(c) $1 \mathrm{wt} \%$ and $2 \mathrm{wt} \%$ of EGDMA are added to participate in the copolymerization reaction, and EGDMA can uniformly cross the monomer MMA. The cross-linked microspheres were prepared to be tight and non-porous, and the surface was smooth and clean. However, when the concentration of EDGMA was increased to $3 \mathrm{wt} \%$ as shown in Figure 8(d) cross-linked structure and surface in cross-linked microspheres, a higher crosslink density results in the appearance of a small number of secondary 


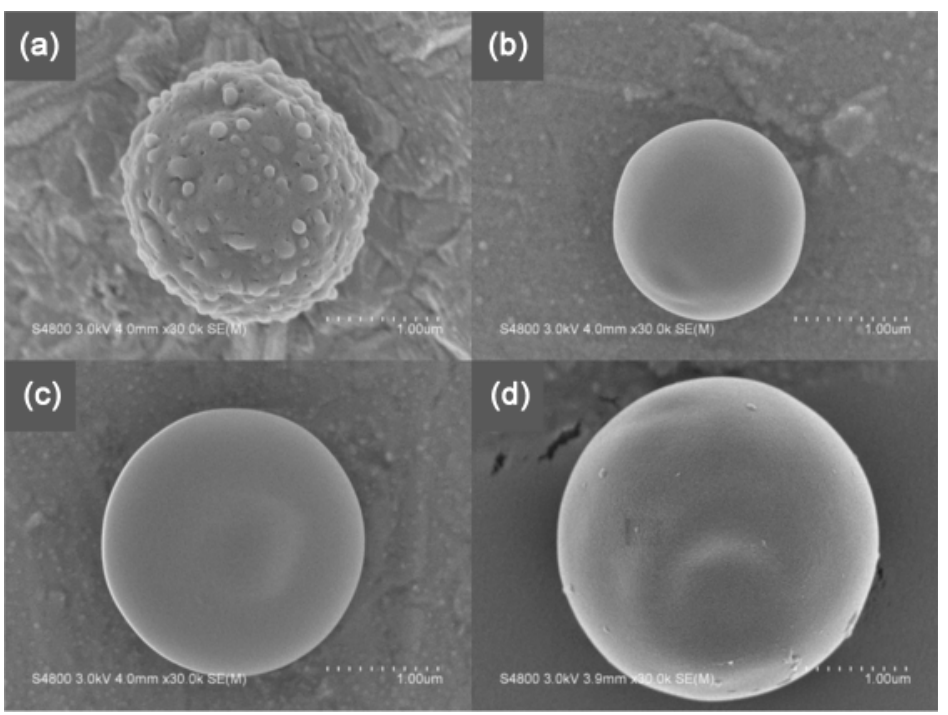

Figure 8. Cross-linked microspheres FE-SEM image prepared with different crosslinker concentrations (a) $0 \mathrm{wt} \%$; (b) $1 \mathrm{wt} \%$; (c) $2 \mathrm{wt} \%$; (d) $3 \mathrm{wt} \%$.

nucleation particles on the surface of the sphere.

Next, adjust the ratio of the polymerization medium to 9/1. Figure 9 shows the FE-SEM image of the crosslinked microspheres prepared by 8 different EGDMA concentrations. Figure 9(a) Microspheres without EGDMA added and Figure 9(b) added 1 wt\% EDGMA cross-linked microspheres, the surface morphology of the two is quite smooth and clean.

Figures 9(c)-(f) are $2 \mathrm{wt} \%$ - $5 \mathrm{wt} \%$ of EGDMA. At the beginning, due to the high crosslink density of crosslinked microspheres, monomeric MMA and oligomers are not uniformly absorbed by the microspheres, resulting in high and low The surface of the microspheres with different undulations and the micropores on the surface, with the increase of the concentration of EGDMA, easily cause the transfer of active radicals in the molecular chain to the monomers in the medium, making the probability of secondary nucleation larger, secondary nucleation The number is increased and the shape is like a myriad of spherical protrusions. If you look closely, you can see in Figures 9(d)-(f) that the growth of the spherical protrusions is from small to large. A small mode is carried out. If the amount of EGDMA added is further increased to $7 \mathrm{wt} \%$, the dent deformation of the crosslinked microspheres can be observed in Figure 9(g), Figure 9(h) because the extremely dense crosslinked structure inhibits the absorption of the oligomer by the microspheres. And the effect of monomer MMA, and the stress effect caused by the difference in crosslink density in the crosslinked microspheres.

\subsection{Particle Size (DLS) Analysis}

Using the laser particle size, the initial addition time, the solvent ratio, and the crosslinking agent addition amount are used as parameters of the particle size analysis. 

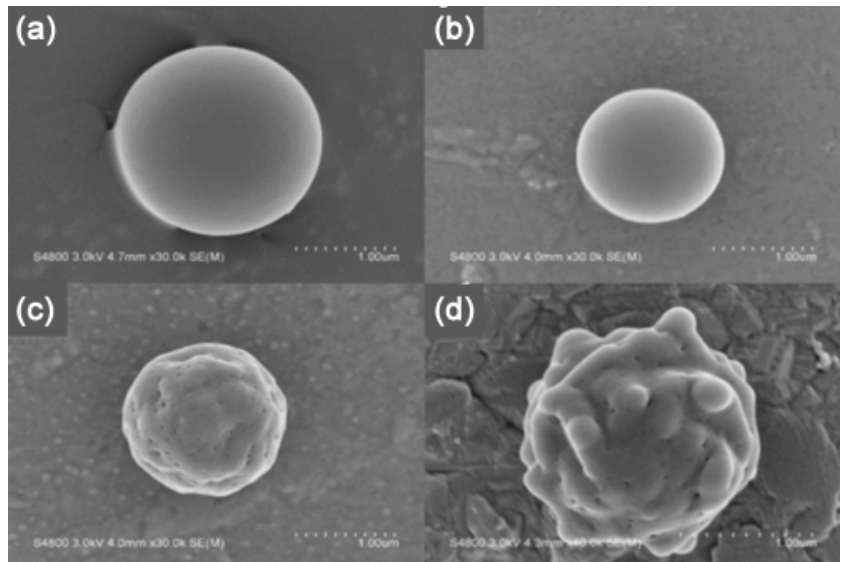

(d)
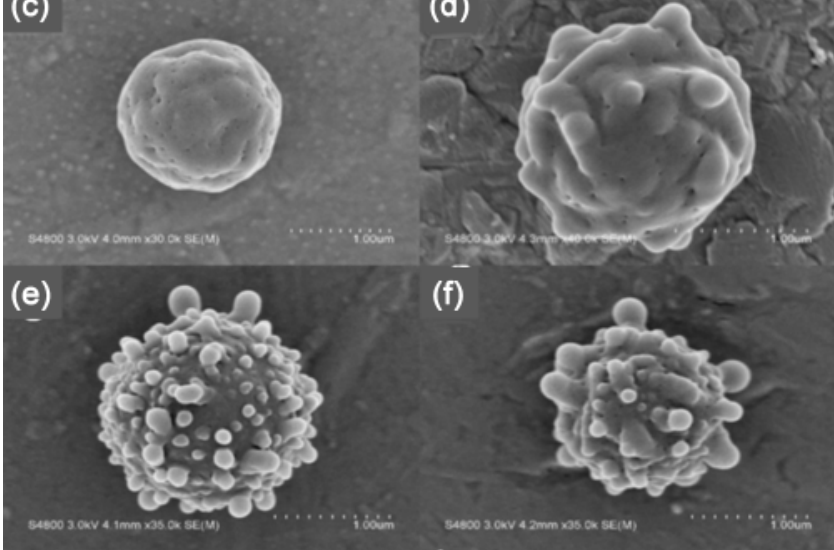

(f)

(g)
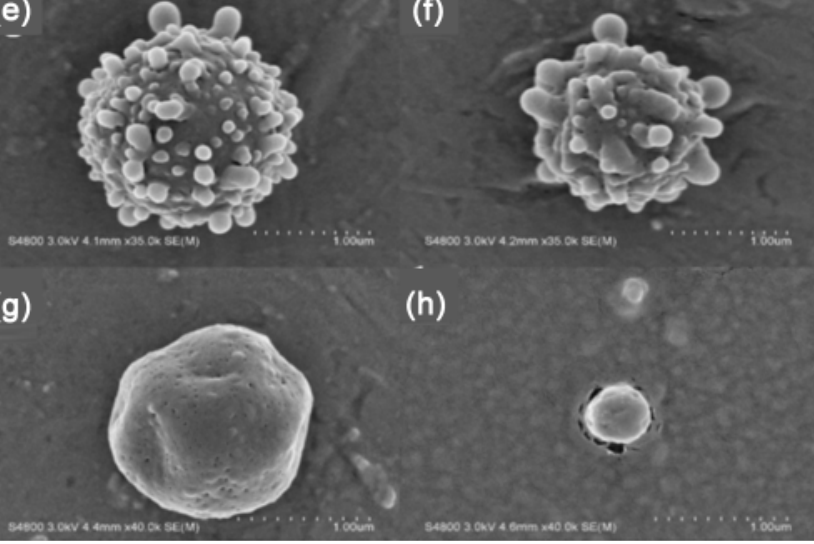

(h)

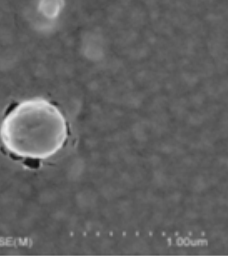

Figure 9. Cross-linked microspheres FE-SEM image prepared by solvent ratio 9/1, different crosslinker concentration. (a) $0 \mathrm{wt} \%$; (b) $1 \mathrm{wt} \%$; (c) $2 \mathrm{wt} \%$; (d) $3 \mathrm{wt} \%$; (e) $4 \mathrm{wt} \%$; (f) $5 \mathrm{wt} \%$; (g) $6 \mathrm{wt} \%$; (h) $7 \mathrm{wt} \%$.

Table 2 compares the PDI values of the laser particle diameters for each parameter.

The average particle size and particle size dispersibility (PDI) of the crosslinked microspheres tend to decrease with the increase of the initial addition time. The crosslinked microspheres prepared at the starting time of 1 hour and 2 hours have a particle size greater than $2 \mu \mathrm{m}$. Corresponding to the FE-SEM analysis discussed in the previous section, the microsphere shape is not complete. Due to the large activity of EGDMA, the addition destroys the balance of the whole system. The particle size of the microsphere is large and the size distribution is wide. After the initial time is increased to 3 hours and 4 hours, the particle size of the crosslinked microspheres is not much different, and the PDI is also reduced because the initial nucleation period of the microspheres has ended and the state in the system is sufficiently stable. The addition of the comonomer does not cause too much interference to the stability of the polymerization system, making the crosslinked microspheres more stable during the growth phase.

According to the ratio of solvent, the particle size and particle size distribution PDI of the microspheres decreased with the increase of the proportion of deionized 
Table 2. (DLS) The initial addition time of the cross-linking agent, the solvent ratio, and the amount of cross-linking agent added as the particle size analysis data.

\begin{tabular}{|c|c|c|}
\hline Initial addition time $(\mathrm{hr})$ & Mean size (um) & PDI \\
\hline 1 & $2.422 \pm 0.949$ & 0.404 \\
\hline 2 & $2.167 \pm 0.327$ & 0.292 \\
\hline 3 & $1.602 \pm 0.125$ & 0.258 \\
\hline 4 & $1.626 \pm 0.346$ & 0.312 \\
\hline Different solvent ratio & Mean size (um) & PDI \\
\hline $10 / 0$ & $1.626 \pm 0.346$ & 0.312 \\
\hline $9 / 1$ & $1.427 \pm 0.909$ & 0.065 \\
\hline $8 / 2$ & $1.109 \pm 0.151$ & 0.025 \\
\hline EGDMA (wt\%) (10/0) & Mean size (um) & PDI \\
\hline 0 & $1.492 \pm 0.160$ & 0.51 \\
\hline 1 & $1.163 \pm 0.526$ & 0.226 \\
\hline 2 & $1.226 \pm 0.547$ & 0.06 \\
\hline 3 & $1.626 \pm 0.346$ & 0.312 \\
\hline EGDMA (wt\%) (9/1) & Mean size (um) & PDI \\
\hline 0 & $1.242 \pm 0.852$ & 0.64 \\
\hline 1 & $1.023 \pm 0.290$ & 0.07 \\
\hline 2 & $1.245 \pm 0.546$ & 0.065 \\
\hline 3 & $1.427 \pm 0.309$ & 0.065 \\
\hline 4 & $1.132 \pm 0.378$ & 0.132 \\
\hline 5 & $1.089 \pm 0.260$ & 0.35 \\
\hline
\end{tabular}

water. The result is that the polarity of methanol and water are 6.6 and 10.2, respectively. Water is good for dispersant PVP. The solvent is a poor solvent for MMA, EGDMA and polymer. Therefore, the water content increases, the polarity of the medium increases, the critical chain length is expected to decrease, the number of crystal nuclei formed and the amount of adsorbed PVP will increase, and the growth of the microspheres will increase. The polymer chain length captured during the process is shorter and the microsphere growth nucleus becomes more stable, so that the crosslinked microspheres have a smaller particle size, a narrower particle size distribution, and a smaller PDI.

The amount of the cross-linking agent added was compared according to the ratio of the solvent. From the solvent ratio of 10/0, the particle size and dispersion of the microspheres showed a downward trend after adding the cross-linking agent. The microspheres of $1 \mathrm{wt} \%$ EGDMA were added, and the particle size decreased from $1.492 \mu \mathrm{m}$ to $1.163 \mu \mathrm{m}$. The addition of EGDMA causes the microsphere structure to change from a linear segment structure to a network cross-linked structure, and the shape becomes looser and more compact. Then, as the concentration of the crosslinking agent increases, the particle 
size of the microspheres increases. It can also be concluded that the PDI of the microspheres with a dispersion of $2 \mathrm{wt} \%$ EGDMA is 0.06 , the particle size is the most uniform, and after the addition of EGDMA increases to $3 \mathrm{wt} \%$, the PDI rises to 0.312 , presumably because of the excessive addition of the crosslinking agent. The surface of the microspheres has a high local crosslink density, and the polymerization system tends to be unstable, resulting in a decrease in particle size uniformity. When the solvent ratio is 9/1, the EGDMA concentration has an effect on the particle size, and the particle size of the microspheres first increases and then decreases. It is concluded that the increase of the crosslinker concentration is more likely to prepare the micro-spheres with a high cross-linking structure. Therefore, the final size of the microspheres depends on the thickness of the shell layer and the concentration of the crosslinker which are crosslinked by the addition of the crosslinker of the microspheres. However, under the assumption that the conversion ratio of the crosslinker and the monomer does not change significantly, $3 \mathrm{wt} \%$ is obviously micro The ball cross-linking density increasing effect is offset by the turning point of the cross-linking agent, which causes the PDI to become larger after that.

In the microsphere diameter data of solvent ratios of $10 / 0$ and $9 / 1$, it was found that the cross-linking agent concentration was in the range of $1 \mathrm{wt} \%$ to 3 wt $\%$, and the particle diameter of the prepared microspheres was less than 10/0. Microspheres, therefore, we can conclude that the amount of cross-linking agent has a certain degree of influence on the size and dispersion of the microspheres, but its influence is still lower than the solvent ratio. [10]

\subsection{Fourier Infrared Spectroscopy (FTIR) Analysis [11]}

Figure 10 is a FTIR spectrum of PMMA microspheres and P (MMA-EGDMA) microspheres [11]. From the spectrum of PMMA microspheres in (a), the $\mathrm{C}=\mathrm{O}$ stretching vibration of the main characteristic peak at $1730 \mathrm{~cm}^{-1}$ can be found, C-O-C stretching vibration of $1150 \mathrm{~cm}^{-1}, 1194 \mathrm{~cm}^{-1}, 1243 \mathrm{~cm}^{-1}$ and $1272 \mathrm{~cm}^{-1}$, with a small peak of $1063 \mathrm{~cm}^{-1}$ on the low wavenumber side of the C-O-C absorption generation, and $1063 \mathrm{~cm}^{-1}$, 988 The polymethyl methacrylate characteristic of $\mathrm{cm}^{-1}$ and $842 \mathrm{~cm}^{-1}$ absorbs the vibrational peak. The vibration of $\alpha-\mathrm{CH} 3$ is shown at $1388 \mathrm{~cm}^{-1}$ and $750 \mathrm{~cm}^{-1}$, and the $\mathrm{CH}$ bond stretching vibration of $2998 \mathrm{~cm}^{-1}$ is the $-\mathrm{CH} 3$ group. The $2952 \mathrm{~cm}^{-1}$ and $2844 \mathrm{~cm}^{-1}$ can be attributed to the $-\mathrm{CH} 2$ group, respectively. The symmetric stretching vibration and asymmetric stretching vibration of the $\mathrm{CH}$ bond, the absorption peak at $1449 \mathrm{~cm}^{-1}$ and $1485 \mathrm{~cm}^{-1}$ is the $\mathrm{CH}$ bond bending vibration of the $-\mathrm{CH} 3$ group, and exists near $3400 \mathrm{~cm}^{-1} \sim 3700 \mathrm{~cm}^{-1}$. The weak absorption band can be attributed to the physically adsorbed moisture- $\mathrm{OH}$ group tensile vibration. As can be seen from the above discussion, the polymer microsphere component is polymethyl methacrylate.

Figure 10(b) is a FTIR spectrum of P (MMA-EGDMA) microspheres, with $\mathrm{CH}$-symmetric stretching vibration and asymmetric stretching vibration of $-\mathrm{CH} 2$ 
group at $2952 \mathrm{~cm}^{-1}$ and $2840 \mathrm{~cm}^{-1}, 1733 \mathrm{~cm}^{-1} \mathrm{C}=\mathrm{O}$ stretching vibration, and COC absorption peaks at $1270 \mathrm{~cm}^{-1}$ and $1149 \mathrm{~cm}^{-1}$, compared with the PMMA microspheres in 10(a), the PMMA characteristic absorption peak intensity can be observed to increase. The results were combined with $1 \mathrm{H}$ NMR to infer whether EGDMA reacted with MMA to form a crosslinked structure, and P (MMA-EGDMA) crosslinked microspheres were successfully synthesized.

\subsection{Functionalized PMMA Microspheres Fourier Infrared Spectroscopy Analysis (FTIR) [12]}

It can be obtained from the FTIR spectrum of Figure 11 that after the microspheres

(a)

PMMA

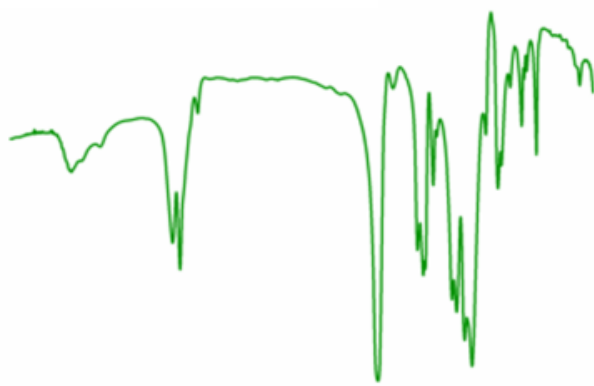

(b)

P(MMA-EGDMA)

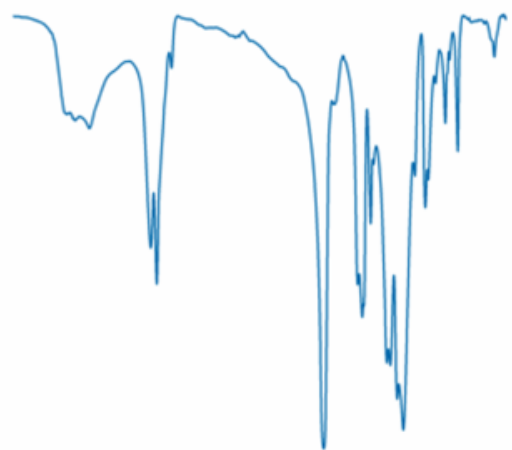

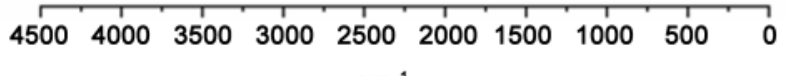

$\begin{array}{llllllllll}4500 & 4000 & 3500 & 3000 & 2500 & 2000 & 1500 & 1000 & 500 & 0\end{array}$ $\mathrm{cm}^{-1}$

Figure 10. Polymeric microspheres FTIR spectrum (a) PMMA; (b) P (MMA-EGDMA).

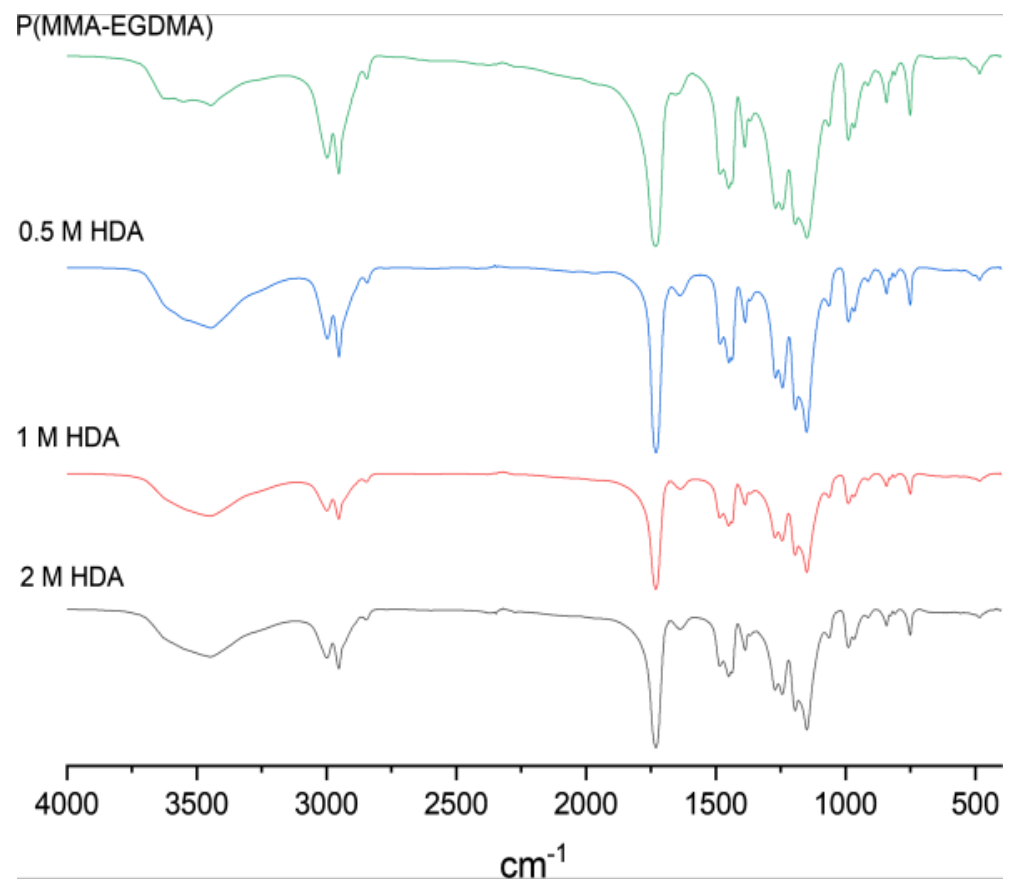

Figure 11. FTIR spectrum of FP (MMA-EGDMA) microspheres. 
are functionalized by hexamethylenediamine, the original P (MMA-EGDMA) microspheres are excited and vibrated at the ester $\mathrm{C}=\mathrm{O}$ at $1733 \mathrm{~cm}^{-1}$, and split into ester stretching vibrations. The absorption peak of the ester with the phthalamide stretching vibration of $1637 \mathrm{~cm}^{-1}$ and $1733 \mathrm{~cm}^{-1}$ was weakened. The absorption band of amine and guanamine was around $3450 \mathrm{~cm}^{-1}$, ranging from $1000 \mathrm{~cm}^{-1}$ to $1300 \mathrm{~cm}$. The COC stretching vibration in the -1 interval and the $\alpha$-CH3 vibration of $1388 \mathrm{~cm}^{-1}$ and $750 \mathrm{~cm}^{-1}$ also weakened after the functionalization of the amide on the surface of the microsphere, which are important indicators of whether or not the hexamethylene diamine is functionalized [13].

\subsection{Hydrogen Nuclear Magnet Resonance Spectroscopy (1H NMR)}

Figure 12 is a ${ }^{1} \mathrm{H}$ NMR spectrum of PMMA microspheres. It can be observed that the chemical shifts at $0.81 \mathrm{ppm}, 0.99 \mathrm{ppm}$, and $1.2 \mathrm{ppm}$ are attributed to the three hydrogen protons of $\mathrm{CH}_{3}$, meta, random, and isotactic. Multiple $\mathrm{CH}_{2}$ hydrogen proton resonance peaks appeared at $1.79 \mathrm{ppm}$ chemical shift, $3.57 \mathrm{ppm}$ represented the hydrogen proton resonance peak of PMMA ester group $\mathrm{OCH}_{3}$, and $3.64 \mathrm{ppm}$ was the $\mathrm{OCH}_{3}$ hydrogen proton resonance peak at the end of the molecular chain.

Figure 13 is a ${ }^{1} \mathrm{H}$ NMR spectrum of $\mathrm{P}$ (MMA-EGDMA) microspheres prepared by adding $2 \mathrm{wt} \%$ EGDMA. The $\mathrm{OCH}_{3}$ hydrogen proton resonance peak of PMMA appeared at $3.57 \mathrm{ppm}$, and the EGDMA end at $5.62 \mathrm{ppm}$ and $6.1 \mathrm{ppm}$ did not participate in the cross-linking reaction. The vinyl hydrogen atom resonance of methyl methacrylate, the broad resonance peak of $4.1 \mathrm{ppm}$ is attributed to the $\mathrm{CH}_{2}$ hydrogen atom of the fully reacted EGDMA, and the chemical shift of $4.3 \mathrm{ppm}$ belongs to the $\mathrm{CH}_{2}$ hydrogen atom resonance of the unreacted EGDMA at one end, thus confirming EGDMA successfully participates in the

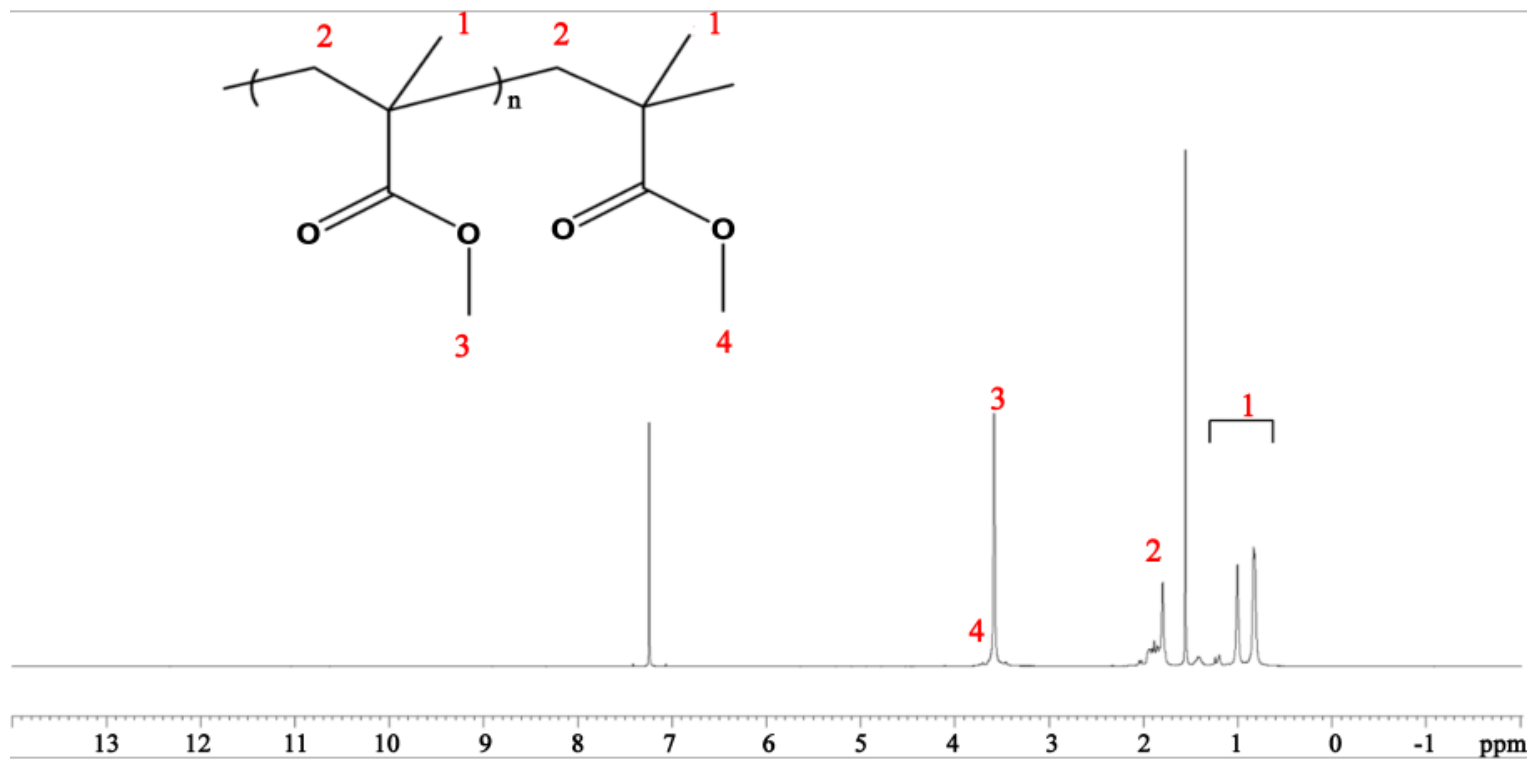

Figure 12. ${ }^{1} \mathrm{H}$ NMR analysis of synthetic PMMA microspheres. 


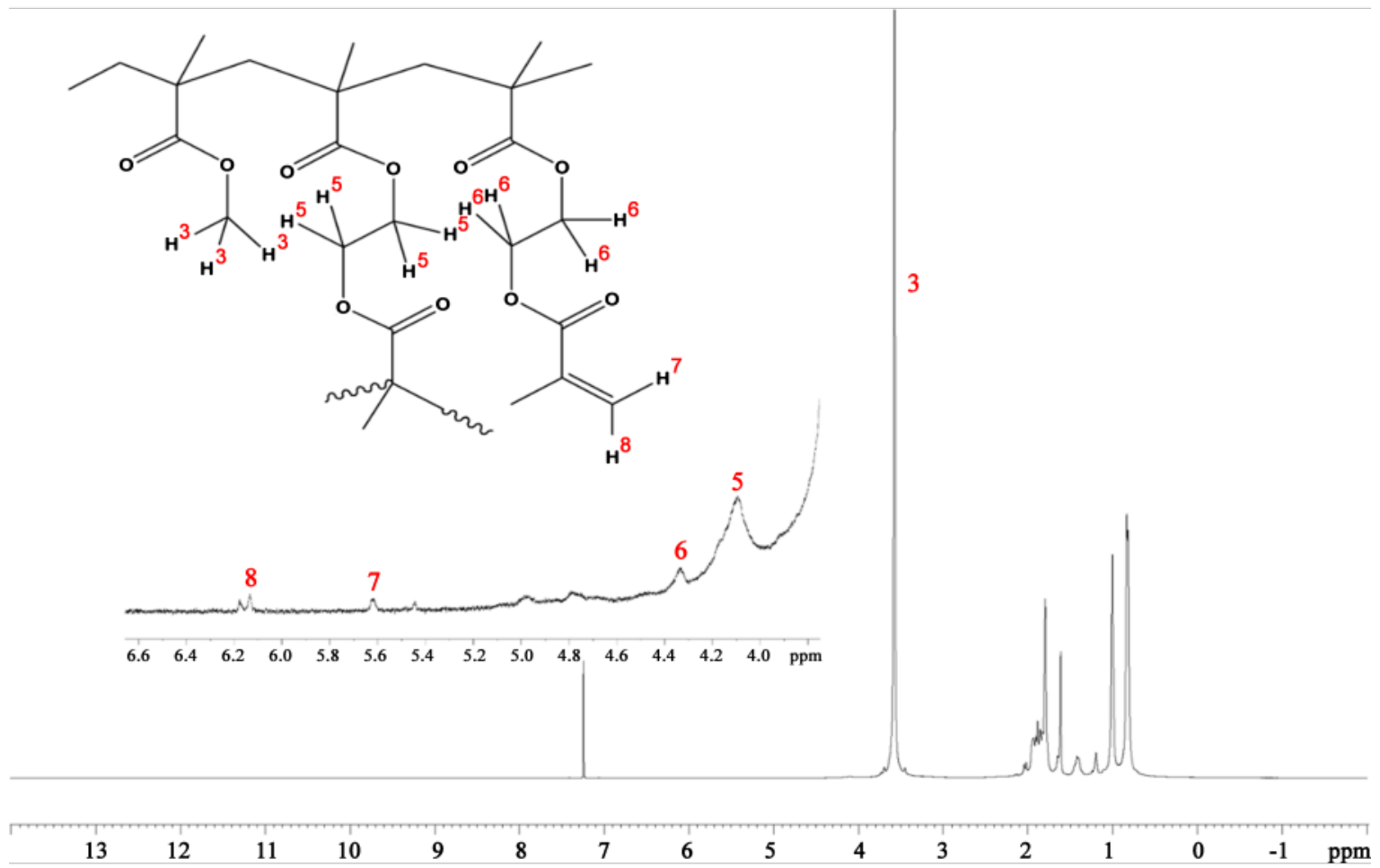

Figure 13. ${ }^{1} \mathrm{H}$ NMR analysis of synthetic P (MMA-EGDMA) microspheres.

reaction to form $\mathrm{P}$ (MMA-EGDMA) microspheres.

\subsection{Thermogravimetric Analyzer (TGA)}

The thermogravimetric loss curves and analytical data tables of PMMA and $\mathrm{P}$ (MMA-EGDMA) microspheres are shown in Figure 14, respectively. The initial pyrolysis temperatures of PMMA and $\mathrm{P}$ (MMA-EGDMA) microspheres are $153.48^{\circ} \mathrm{C}$ and $145.41^{\circ} \mathrm{C}$, respectively. Both are almost completely degraded after $440^{\circ} \mathrm{C}$.

Compared with PMMA microspheres, the initial cracking temperature of $\mathrm{P}$ (MMA-EGDMA) microspheres is about $8^{\circ} \mathrm{C}$ lower. Before $50 \%$ weight loss point, the $\mathrm{P}$ (MMA-EGDMA) weight loss curve is also lower than PMMA. Because there is incomplete cross-linking in the network structure formed by MMA and EGDMA, many short-chain branches appear on the side of the macromolecular segment, so there are more unsaturated segment ends, and the number of free radical transferable places increases, causing this to happen.

\subsection{Differential Scanning Thermal Analysis (DSC)}

Figure 15 and Figure 16 are the primary and secondary temperature rise curves of PMMA and P (MMA-EGDMA) microspheres respectively. The PMMA microspheres have a heat absorption and release peak near the temperature rise curve of $60^{\circ} \mathrm{C}$, and the exothermic area of $114.85^{\circ} \mathrm{C}$ can be speculated or not. The reaction monomer was related to evaporation at a temperature higher than the Tg point, and the secondary heating curve showed no significant exothermic 


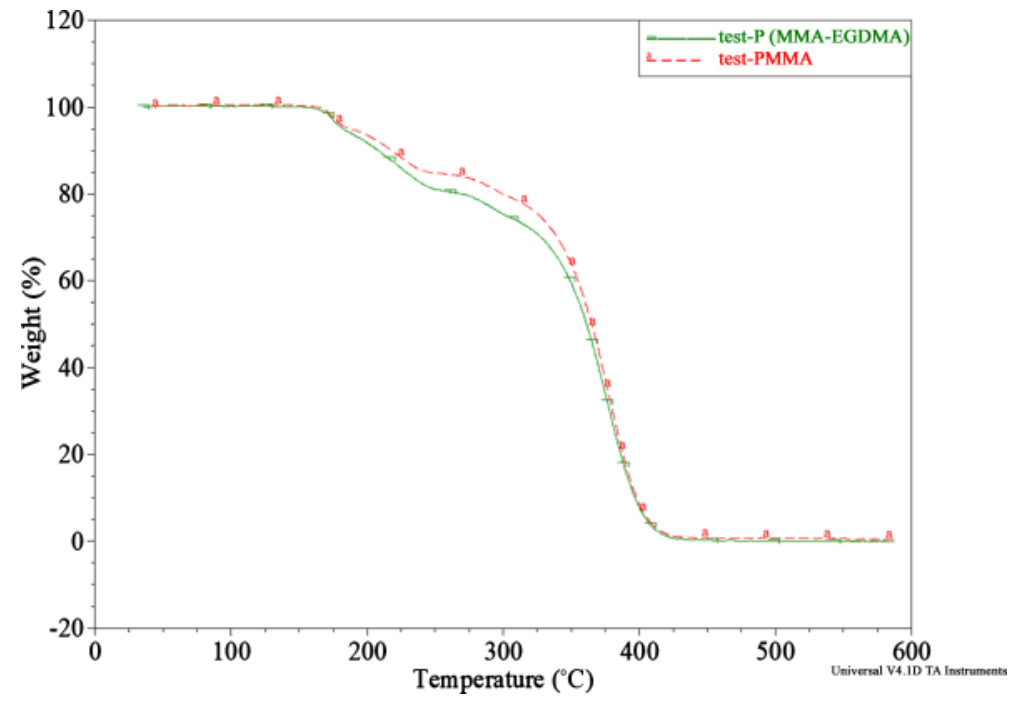

Figure 14. Microsphere thermogravimetric loss curve.

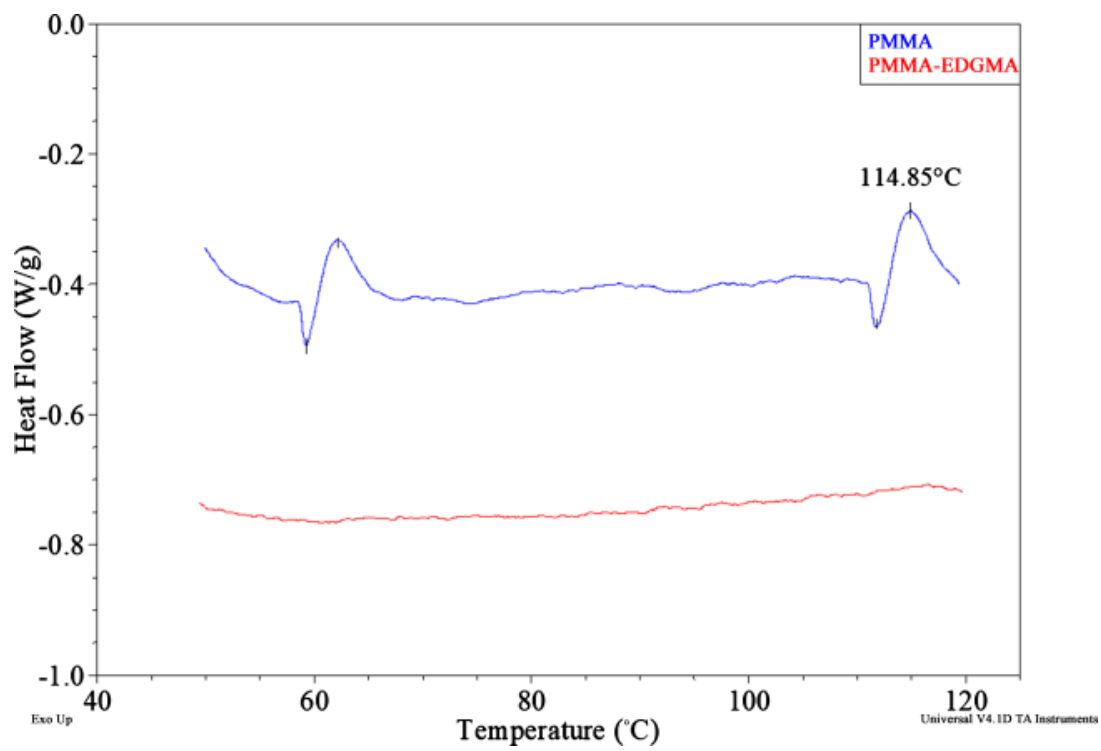

Figure 15. Microsphere one-time heating analysis curve.

peak. From the DSC analysis curve of P (MMA-EGDMA) microspheres, it can be observed that there is no obvious glass transition zone in the crosslinked microspheres, and the curves are smooth and there is not much change, which is attributed to the molecular structure of the microspheres. It is difficult to carry out thermal motion. Therefore, it can be inferred that the micro-crosslinked structure leads to the absence of Tg points. It is a typical amorphous structure curve. It can also be inferred from the one-time heating curve that the addition of a crosslinking agent can inhibit the amount of unreacted monomers in the microspheres.

\subsection{Energy Dispersive X-Ray Spectroscopy (EDS) Analysis}

Figure 17 and Figure 18 show the EDS analysis data of FP (MMA-EGDMA) 


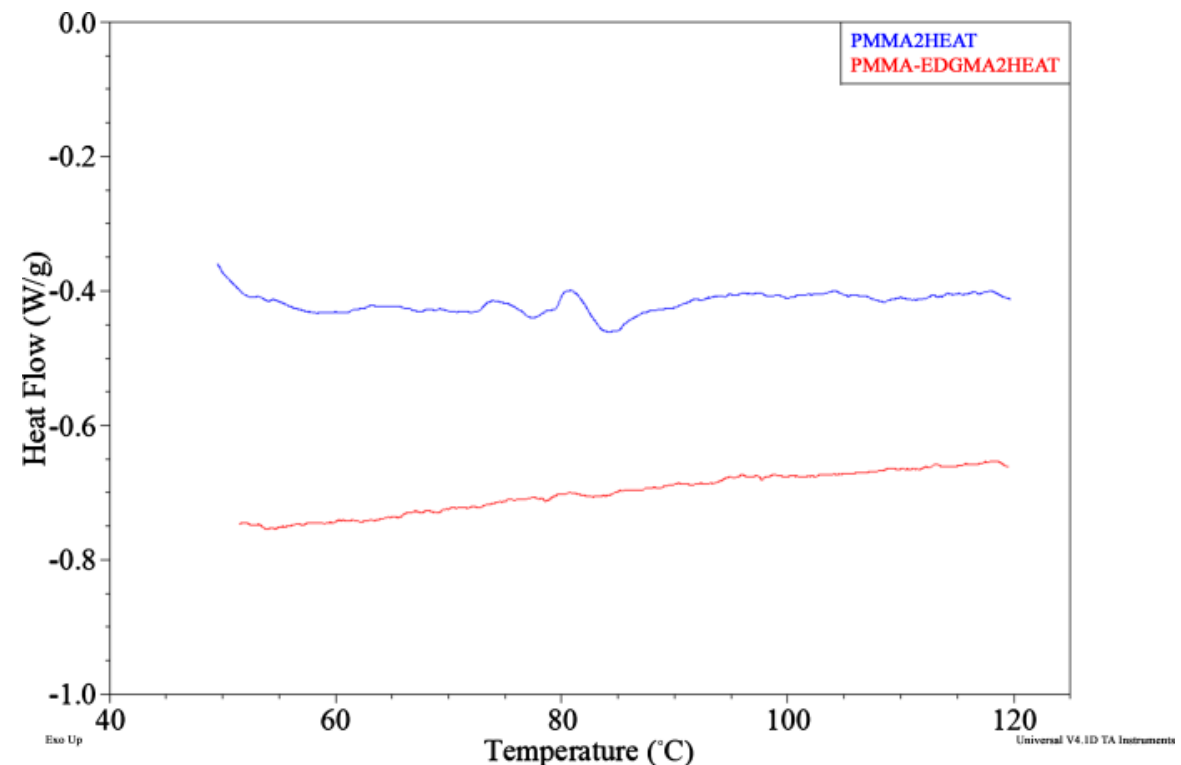

Figure 16. Microsphere secondary heating analysis curve.

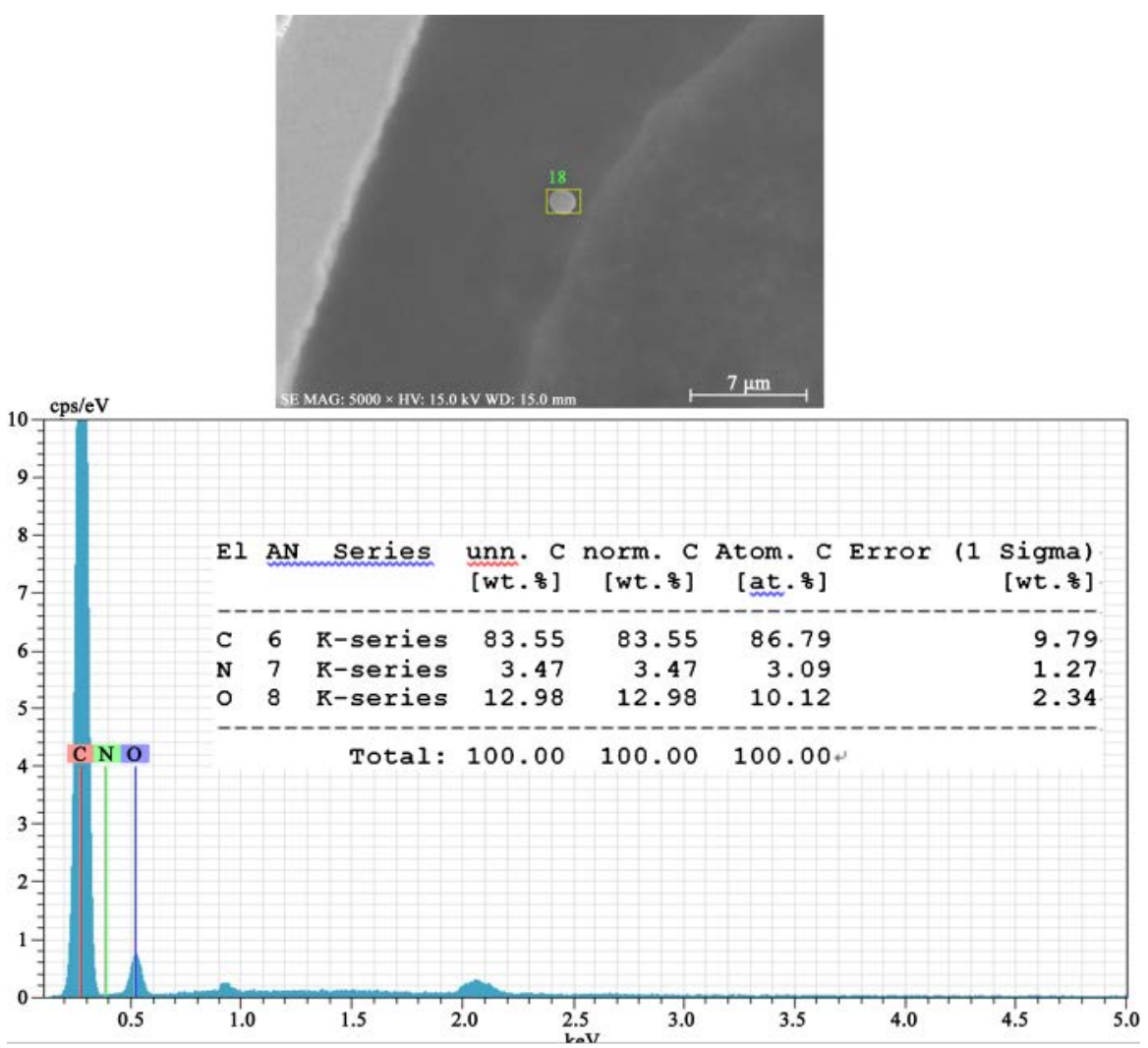

Figure 17. Analysis of EDS Spectrum of 0.5 M Hexamethylenediamine FP (MMA-EGDMA) Microspheres.

microspheres prepared at $0.5 \mathrm{M}$ and $1 \mathrm{M}$, respectively. All of them were tested by a single microsphere at the yellow frame in a FE-SEM image taken at $5.0 \mathrm{~K}$ magnification. It can be obtained that the molecular weight of the microspheres to which $1 \mathrm{M}$ hexamethylenediamine is added is $12.41 \mathrm{wt} \%$, which is higher than 


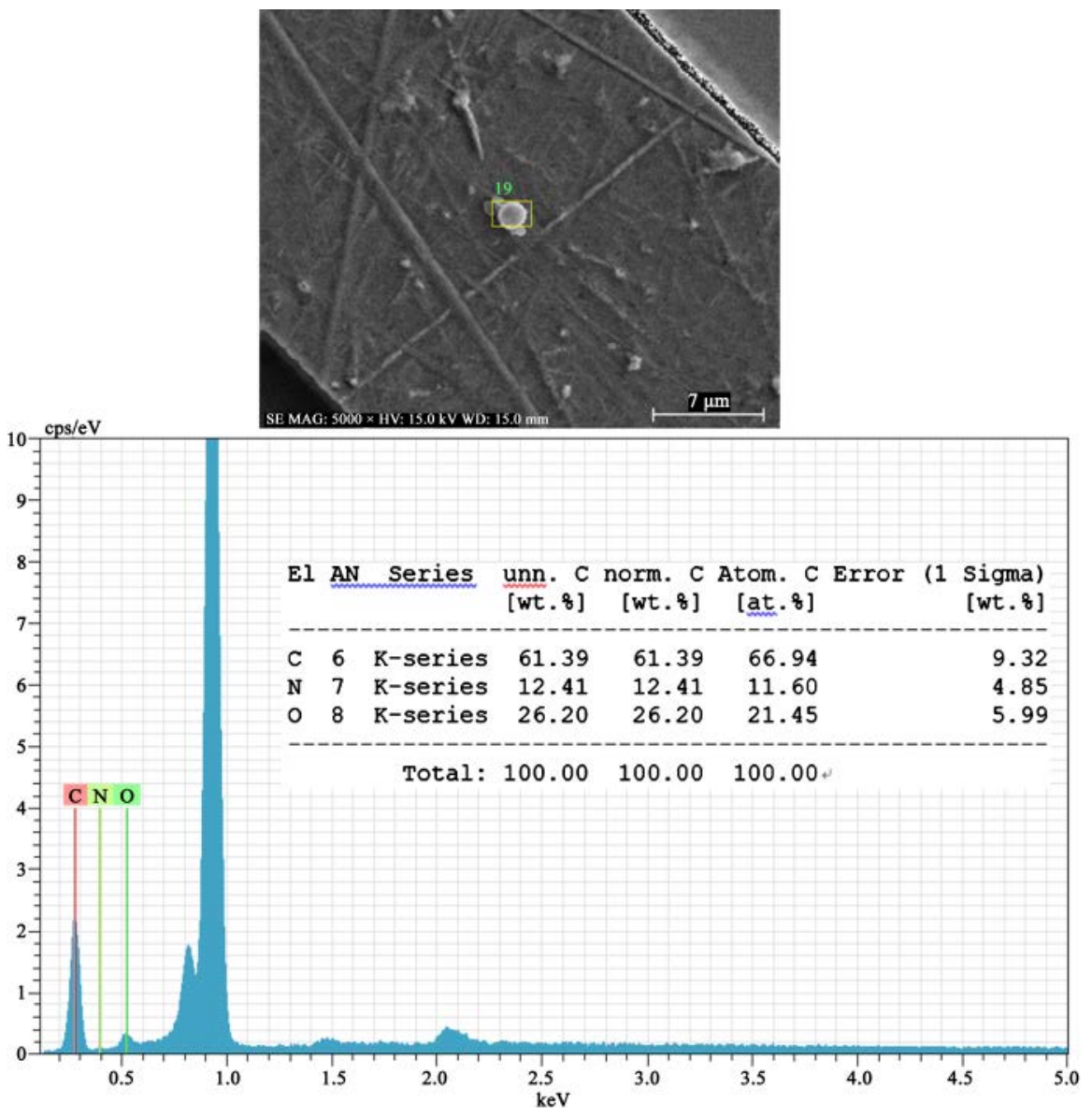

Figure 18. $1 \mathrm{M}$ hexamethylenediamine FP (MMA-EGDMA) microsphere EDS spectrum analysis.

the $3.47 \mathrm{wt} \%$ of the $0.5 \mathrm{M}$ microspheres, indicating that more ester groups on the surface of the microspheres react with hexamethylenediamine. Amidoxime, this result also confirmed that hexamethylenediamine was successfully grafted on the surface of the microspheres, and the amine was immobilized on the outside of the microspheres, and the amino-functionalized microspheres were successfully prepared.

\subsection{Effect of Dyeing pH on Microsphere Dyeing Performance}

Figure 19 and Table 3 show the color intensity (K/S) change trend and color detection data of FP (MMA-EGDMA) microspheres dyed with black mixed acid dyes at different $\mathrm{pH}$ values. It can be found that with the increase of potential of hydrogen, the K/S values showed a downward trend with the highest $\mathrm{K} / \mathrm{S}$ value at $\mathrm{pH}$, reaching 2.7068. Under acidic dyeing conditions, the amine group $\left(-\mathrm{NH}_{2}\right)$ on the surface of FP (MMA-EGDMA) microspheres adsorbed protons $\left(\mathrm{H}^{+}\right)$, the cationization is converted to positively charged $\mathrm{NH}_{3}^{+}$, and the 1:2 type acid dye central metal ion chelating group has been occupied by the lone pair of electrons on the chromophoric group of the dye chromophore and the azo chromophore. The ball is chelated and is mainly dyed by ionic bonds. Therefore, 


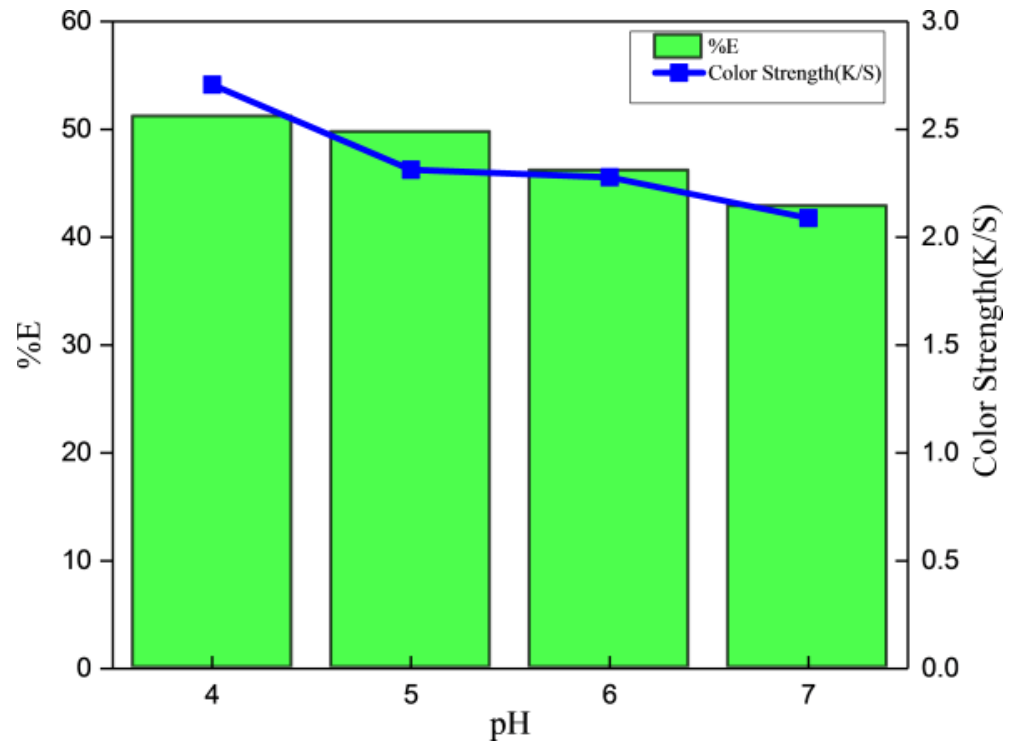

Figure 19. Trends in color intensity of dyed microspheres and depletion rate at different $\mathrm{pH}$ values.

Table 3. Apparent concentration of Color detection value and CIE $L^{*} a^{*} b^{*}$.

\begin{tabular}{ccccc}
\hline $\mathrm{pH}$ & $\mathrm{L}^{*}$ & $\mathbf{a}^{*}$ & $\mathbf{b}^{*}$ & $\Delta \mathrm{E}$ \\
\hline 4 & 47.58 & 1.6 & 5.91 & 42.66 \\
5 & 48.73 & 1.24 & 2.55 & 44.39 \\
6 & 49.38 & 1.55 & 1.35 & 60.97 \\
7 & 47.96 & 1.57 & 1.21 & 70.87 \\
\hline
\end{tabular}

it is inferred that the number of cationized amine groups on the surface of the microspheres is higher at $\mathrm{pH} 4$, so that more dyed seats can be combined with the acid dye, but the $\mathrm{pH}$ can be found corresponding to Table 3. At 4 o'clock, the dyed microspheres showed a blackish yellow color with a b value of 5.91, which was at least 2 times higher than the other $\mathrm{pH}$ values. The reason was presumed to be the mixed dyeing solution of the blending, the affinity of the yellow acid dye and the microspheres. Larger, dyeing problems between the dyes, resulting in this problem is highlighted at lower $\mathrm{pH}$ [14] [15]. Figure 19 shows the dyebath depletion rate (\% $\mathrm{E}$ ) of dyed microspheres dyed at different dyeing $\mathrm{pH}$ values. The dye depletion rate decreases with increasing $\mathrm{pH}$ value, which proves that the dye does generate ions with the microspheres. Bonding, but the percentage of depletion rate is not high at around $50 \%$. It may be because the dye concentration is too high, and the dye molecules are more than the microspheres dyed the seats, so that the dye cannot be completely combined with the microspheres.

\subsection{Cold Field Emission Scanning Electron Microscopy (FE-SEM) Analysis and High Resolution Transmission Electron Microscopy (HRTEM) Analysis}

Figure 20 is a TEM image of P (MMA-EGDMA) microspheres. It can be confirmed 


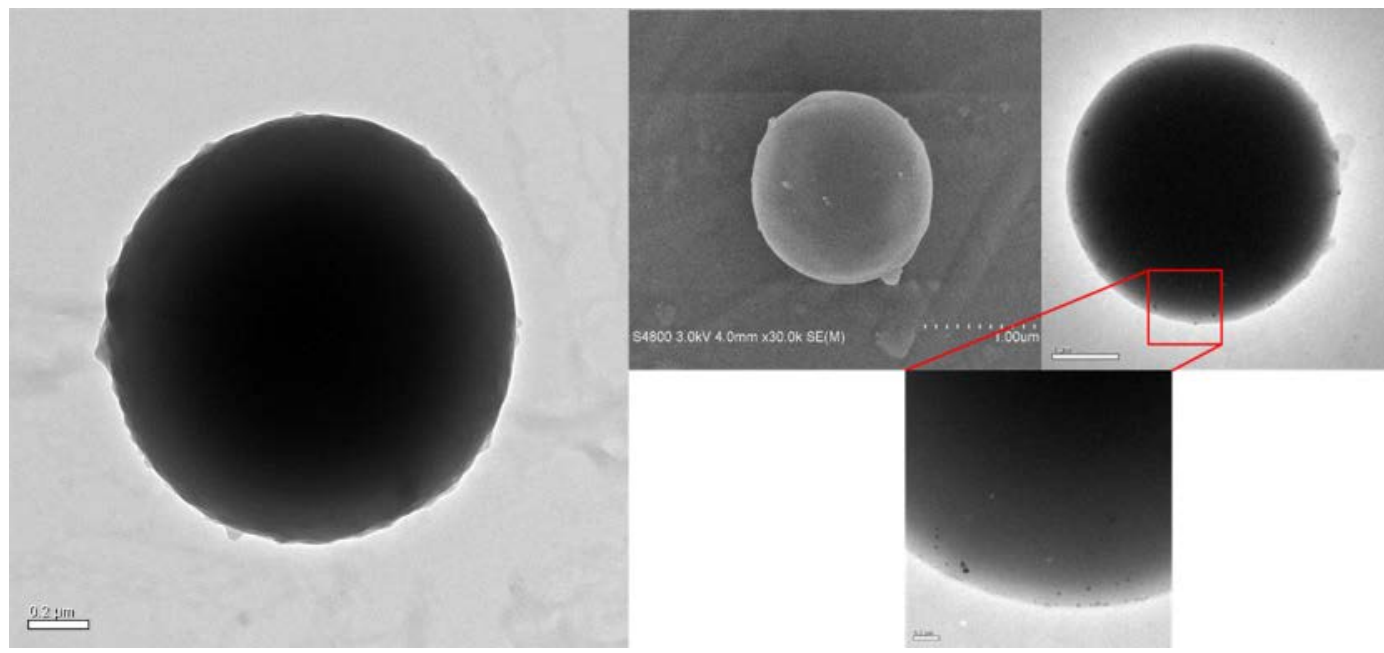

Figure 20. HRTEM image of P (MMA-EGDMA) microspheres, FE-SEM image, TEM image and magnified view of the red frame.

from the figure that the P (MMA-EGDMA) microspheres have a solid structure without the appearance of hollow structures or voids. Figure 20 shows the dyeing at $130^{\circ} \mathrm{C}$. The microspheres FE-SEM and HRTEM images were observed from FE-SEM. The dyed black FP (MMA-EGDMA) microspheres had slightly uncleaned dye particles on the surface, and the TEM image was also observed. It can be observed that there is basically no change in the appearance of the microspheres. It can be inferred that the combination of the dye and the microspheres does not cause significant damage to the microspheres. Therefore, it can be proved that the preparation of black spacer microspheres using acid dyes in this study is extremely the way of potential.

\section{Conclusion}

In this study, FP (MMA-EGDMA) microspheres with uniform particle size were prepared by dispersion polymerization. The best polymerization parameters were EGDMA initial addition time of 4 hours and the added concentration was $2 \mathrm{wt} \%$. The surface of the cross-linked microspheres was smooth and round, and the average particle size was $1.226 \mu \mathrm{m}$. FTIR and H NMR analysis confirmed that EGDMA did react with MMA monomer to form a cross-linked network structure, and successfully synthesized P (MMA-EGDMA) micro. The ball is dyed with a black dye mixed with a 1:2 type metal complex acid dye. According to the dyeing conditions, $\mathrm{pH} 5$ has the best color intensity, the $\mathrm{L}$ value is the lowest, and the $\mathrm{a}$ and $\mathrm{b}$ values are closest to 0 . The color is closest to pure black. Finally, FE-SEM and HRTEM analysis confirmed that the P (MMA-EGDMA) microspheres synthesized in this study were solid, and did not affect the surface appearance after dyeing. [16] [17].

\section{Conflicts of Interest}

The authors declare no conflicts of interest regarding the publication of this paper. 


\section{References}

[1] Arshady, R. (1992) Suspension, Emulsion, and Dispersion Polymerization: A Methodological Survey. Colloid and Polymer Science, 270, 717-732. https://doi.org/10.1007/BF00776142

[2] Liu, H.B. and Wang, C.C. (2008) Preparation of Mono-Dispersed Fluorescent PMMA/GMA/DVB Polymer Microspheres with Different Sizes. Acta Chimica Sinica, 66, 1269-1273.

[3] Wu, H.-T., Ding, C.-C. and Chen, K.-J. (2013) Preparation of Monodispersed PMMA Particles and Composite Particles Containing Pigment Green 36 by Dispersion Polymerization. Journal of the Taiwan Institute of Chemical Engineers, 44, 691-699. https://doi.org/10.1016/j.jtice.2012.12.026

[4] Duan, G., Zhang, C., Li, A., Yang, X., Lu, L. and Wang, X. (2008) Preparation and Characterization of Mesoporous Zirconia Made by Using a Poly(Methyl Methacrylate) Template. Nanoscale Research Letters, 3, 118-112. https://doi.org/10.1007/s11671-008-9123-7

[5] Sivakumar, M. and Panduranga Rao, K. (2000) Synthesis and Characterization of poly(methyl methacrylate) Functional Microspheres. Reactive \& Functional Polymers, 46, 29-37. https://doi.org/10.1016/S1381-5148(00)00033-X

[6] Lee, K.-C. and Lee, S.-Y. (2008) Preparation of Highly Cross-Linked, Monodisperse Poly(methyl methacrylate) Microspheres by Dispersion Polymerization; Part II. Semi-Continuous Processes. Macromolecular Research, 16, 293-302. https://doi.org/10.1007/BF03218520

[7] Lee, K.-C. and Lee, S.-Y. (2007) Preparation of Highly Cross-Linked, Monodisperse Poly(Methyl Methacrylate) Microspheres by Dispersion Polymerization; Part I. Batch Processes. Macromolecular Research, 15, 244-255. https://doi.org/10.1007/BF03218783

[8] Peng, B., van der Wee, E., Imhof, A. and van Blaaderen, A. (2012) Synthesis of Monodisperse, Highly Cross-Linked, Fluorescent PMMA Particles by Dispersion Polymerization. Langmuir, 28, 6776-6785. https://doi.org/10.1021/la301288r

[9] Morgen, T.O., Krumova, M., Luttikhedde, H. and Mecking, S. (2018) Free-Radical Dispersion Polymerization of Ethylene with Laponite to Polyethylene-Clay Nanocomposite Particles. Macromolecules, 51, 4118-4128.

https://doi.org/10.1021/acs.macromol.8b00440

[10] Park, S.H., Kim, J., Lee, W.-E., Byun, D.-J. and Kim, M.H. (2017) One-Step Synthesis of Hollow Dimpled Polystyrene Microparticles by Dispersion Polymerization. Langmuir, 33, 2275-2282. https://doi.org/10.1021/acs.langmuir.6b04428

[11] Tan, J., Li, X., He, J., Xu, Q., Zhang, Y., Dai, X., Yu, L., Zeng, R. and Zhang, L. (2017) Carboxyl-Functionalized Polymeric Microspheres Prepared by One-Stage Photoinitiated RAFT Dispersion Polymerization. Polymers, 9, 681-694. https://doi.org/10.3390/polym9120681

[12] Chen, B., Liu, J., Fu, S., Zhang, W. and Wang, J. (2013) Synthesis and Characterization of Functional PMMA Micrspheres with Carboxyl Surface. New Chemical Materials, 41, 92-94.

[13] Usman, M., He, L., Wang, Y.-F., Yu, B. and Cong, H.-L. (2018) Magnetic Poly(PMMA-EGDMA) Nanospheres Prepared by Miniemulsion Polymerization. Ferroelectrics, 529, 168-173. https://doi.org/10.1080/00150193.2018.1458536

[14] Fang, K. and Ren, B. (2014) A Facile Method for Preparing Colored Nanospheres of Poly(Styrene-co-Acrylic Acid). Dyes and Pigments, 100, 50-56. 
https://doi.org/10.1016/j.dyepig.2013.07.021

[15] Hou, Y., Zhao, T., Shi, Y., Fan, J., Zheng, R., Zhang, Y. and Gu, Q. (2018) Surface Modification of Carbon Black to Facilitate Suspension Polymerization of Styrene And Carbon Black. Journal of Applied Polymer Science, 135, 46387.

https://doi.org/10.1002/app.46387

[16] Elshereksi, N.W., Mohamed, S.H., Arifin, A. and Ishak, Z.A.M. (2014) Thermal Characterisation of Poly(Methyl Methacrylate) Filled with Barium Titanate as Denture Base Material. Journal of Physical Science, 25, 15-27.

[17] Shim, S.E., Yang, S., Jung, H. and Choe, S. (2004) Thermally Robust Highly Crosslinked Poly(Methylmethacrylate-co-Divinyl Benzene) Microspheres by Precipitation Polymerization. Macromolecular Research, 12, 233-239.

https://doi.org/10.1007/BF03218393 